\title{
The dynamics of the photochromic effect in tungsten trioxide
}

\author{
C. Bechinger, D. Ebner, S. Herminghaus and P. Leiderer
}

Fakultä.t, für Plıysik, Lniversität. Konsta.nz, 78431 Konstanz, Cirrmany

\begin{abstract}
Based on the current. model of the photochromic effert in thin films of tungsten trinxide, we propose a simple nonlinear rate equation which accounts very well for the details of the temporal hehaviour of the ahsorbance of the films. This strongly supports our model.
\end{abstract}

Tungsten trioxide has been well known as a chromogenic material for a long time $e^{1,2}$. Thin films of this material can be reversibly switched between a transparent and a st.rongly colored (blue) state. This makes it most promising for technological applications such as 'smart windows' or displays. While coloration experiments are usually performed in electrochemical cells, coloration can also be achieved by irradiation with ultraviolet (UV) light 1,3 . This so-called photochromic effect has been known as well, but a microscopic understanding was lacking. Recently, we reported on time-resolved measurements of the absorptivity of thin $(\approx 100 \AA)$ evaporated tungsten trioxide films using optically excited surface plasmons ${ }^{4,5}$. By observing the behaviour of the subtle coloration luring and after UV-irradiation led to considerable progress towards a detailed understanding of the photochromic effect. In this paper, we take another step in this direction proposing a nonlinear rate equation derived from our model and demonstrating its accordance with the experi. ment.

It has been suggested that the photochromic effect in tungsten trioxide is intimalely connerted to the photo-induced decomposition of the incorporaled water, which results in the simul-

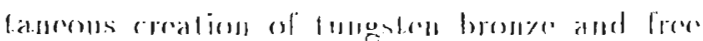
oxygen ${ }^{4}$. It was found that the dynamics of both the coloration and the subsequent spontaneous bleaching (after the irradiation is terminated) is strongly influenced by the oxygen concentration in the sample. In this letter wo want to adress the details of the spontaneous bleaching process.
First, we give a summary of the phenomenology of spontaneous bleaching in oxygen atmosphere. As shown in fig.1, the absorptivity of the sample (as a function of time) can be very well fitted by the sum of two exponential functions,

$$
\alpha=A_{1} e x p^{-t / \tau_{1}}+A_{2} e x p^{-t / \tau_{2}}
$$

where the time constants $\tau_{1}$ and $\tau_{2}$ are on the order of $20 \mathrm{sec}$ and $130 \mathrm{sec}$, respectively, the latter rlepending considerably on the preparation conditions of the sample. Furthermore, the amplitude $A_{2}$ is strongly dependent on the partial pressure of oxygen in the surrounding atmosphere, while for $A_{1}$ no significant dependence is found.

Since it is known that evaporated tungsten t.rioxide films have a porous structure consisting of very small crystallites ${ }^{6,7}$, it is natural to consicler the color center's wilhin the crystallites and those at the inner surfaces separately. 'The volor conters at the inner surfaces will be able to exclange oxygen witl the surremeling atmosphere and will then be associated with the antplitude $A_{2}$, while those localerel within the erystallites will decay by recombination with the interstitial oxygen created simultancously with them ${ }^{4,5}$.

For the latter, the rate equation reads

$$
\partial_{\ell} \int_{c}=-p \int_{c}
$$

where $f_{c}$ denotes the density of color centers located within the crystallites and $p$ is their recombination probability with the corresponding inlerstitial oxygen. Accordingly, the decay will follow a simple exponential, with both time con- 


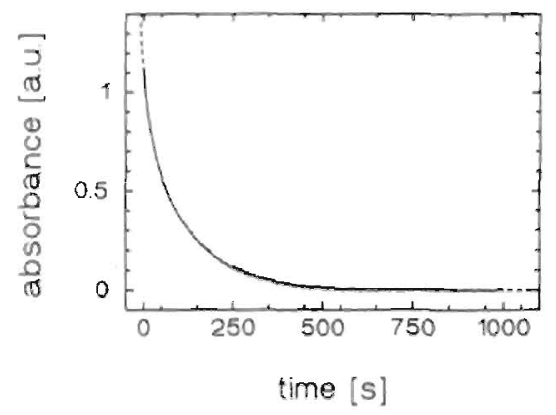

Figure 1: The decay of the coloration, after the UV arradiation is torminatcd. The fitted curve (the sum of two exponentials, dashed line) fits the data so well that it can hardly be distinguished from the data points. The deviations are, however, significant and are accounted for by a nonlinear rate equation (see text).

stant and amplitude being independent of the oxygen partial pressure. This can be identified with the fast decay $A_{1} e x p^{-t / \tau_{1}}$ (first term on the rhs. of eq. 1 , in accordance with the experiment. However, the decay of those color centers which can exchange oxygen with the atmosphere is expected to be more complicated. Denoting the density of the color centers at the inner surfaces by $\int_{s}$ ind the density of free oxygen in the sample by $n$, we can immediately

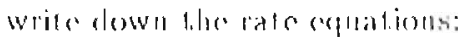

$$
\begin{aligned}
\partial_{t} f_{s} & =-a \int_{s} n \\
\partial_{t} n & =-\frac{1}{2} a f_{s} n-b n-c
\end{aligned}
$$

where a denotes the recombination probability (color center - oxygen), b is the probability for the oxygen to leave the film and $c$ describes the oxygen uptake from the surrounding atmosphere. Here we have assumed that the diffusive transport of oxygen in the sample is fast enough for $n$ to be treated as spatially constant. The factor $\frac{1}{2}$ in the second equation stems from the fart that one oxygen atom can bleach two color centers ${ }^{\mathbf{4}}$.

For $t \rightarrow \infty$, the dynamical system given by eq. 3 will tend to the stable fixed point $\left(\int_{s}, n\right)=(0, c / b)$. In the vicinity of the fixed point, which corresponds to a blearhed state $\left(f_{s}=0\right)$, the decay will be exponential, as in eq. 1 with $\tau_{2}=b / a c$. It seems straightforward to test this prediction by measuring the dependence of $\tau_{2}$ from the oxygen pressure. However,

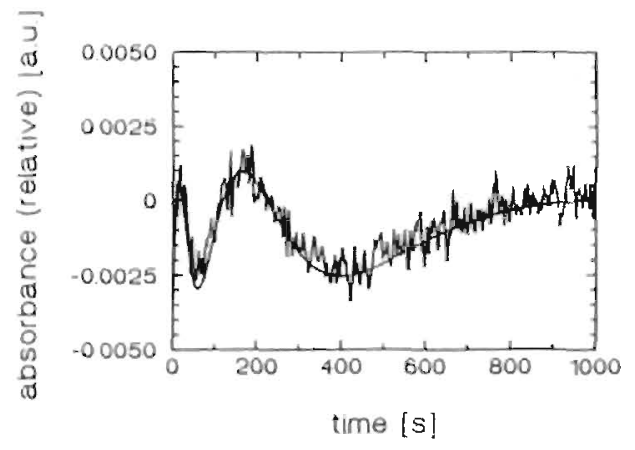

Figure 2: The deviation of the absorbance from the double exponential fit. A pronounced oscillation is clearly present well above noise tevel. The numerical solution of eq. $\$$ is represented by the smooth solid line, which closely follows the observed oscillation.

this turns out to be difficult since the constant $c$ also depends on the adsorption behaviour of the oxygen on the $\mathrm{WO}_{3}$ surface, which is hard to control.

We thus chose to concentrate on the deviations from the exponential behaviour, which are to be expected farther away from the fixed point, due to the nonlinearity of the system. For this purpose we have subtracted the double exponential fit according to eq. 1 from the data. This is shown in fig.2, where this difference is plotted on the same time scale as in fig.1. An oscillation in the absorptivity (i.c., the color center density $f=f_{c}+f_{s}$ ) is clearly visible well above noise level.

Next we performed a numerical calculation of the solutions of eq. 3 and varied the parameters so as to obtain a good fit to our data. All three parameters $(a, b$ and $c)$ turued out to be on the order of $10^{-2} \mathrm{sec}^{-1}$, which is a reasonable time seale for oxygen exchange between it mosphere and metal oxide surfaces ${ }^{8}$. Then we subtracted the double exponential (obtained by fitting the experimental data) from the numerical solution. This difference is shown as the smooth solid line in fig.2. The agreement of theory and experiment is remarkable.

This gives us confidence that our simple oxygen exchange model actually contains the physical processes relevant for the photochromic effect in thin films of $\mathrm{WO}_{3}$. The observed oscillation shown in fig. 2 and the similarity of eq. 3 to the Lotka-Volterra model ${ }^{9}$ raises the 
interesting question whether and under what. circumstances these films can be driven into dynamically unstable states, exhibiting spatio- temporal chaos as other nonlinear systems ${ }^{9,10}$.

This work was supported by the Zentrum II Finergieforschung.

\section{References}

I S.K.Deb; Phil. Mag. 27, 801 (1973).

2 K'Bange. 'T.Ciambke; Aderned Mal. 1,10 (1990).

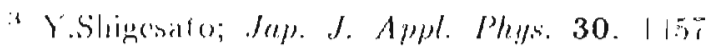
(1991).

I C. Bechinger, G.Oefinger, S.Herminghaus, P.leiderer; will be published in I. Appl. Phys. (oct. 1993).

5 (..Bechinger, S.Herminghaus, P.Leiderer; accepted for publication in Thin Solid riblms.

${ }^{6}$ M.Shiojiri, T'Miyano, C.Kaito; Jap. J. Appl. Phys. 17, 567 (1978).
7 C.Kaito, T.Shimizu, Y.Nakata, Y.Saito; Jap. J. Appl. Phys. 24, 117 (1985).

${ }^{8}$ K.Hauffe, Th.Wolkenstein, edts.: 'F.lectronic Phenomena in Chemisorption and Catalysis on Semiconductors; Symp.Proc. Moscow, July 24, 1968 (W.deGruyter, Berlin 1969).

9 H.Haken: 'Synergetics' (Springer, Berlin 1977).

10 J.Lauterbach, G.Hads, H.H.Rotermund, G.Erti; Surf.Sci. 294, 116 (1993). 